\title{
New Gene Profiling in Determination of Breast Cancer Recurrence and Prognosis in Iranian Women
}

\author{
Seyed Mohammad Poorhosseini ${ }^{1}$, Mohammad Hashemi ${ }^{2}$, Nasrin Alipour Olyaei ${ }^{6}$, \\ Amir Izadi ${ }^{3}$, Elham Moslemi ${ }^{4}$, Zeinab Ravesh $^{6}$, Feyzollah Hashemi-Gorji $^{6}$, Hamid \\ Reza Kheiri ${ }^{5}$, Vahid Reza Yassaee ${ }^{1,6 *}$
}

\begin{abstract}
Breast cancer (BC) is the second most common cancer in the world and by far the most frequent cancer among women, with an estimated 1.67 million new cancer cases diagnosed in 2012 (25\% of all cancers). Polygene expression analysis is used to predict the prognosis and determine the most appropriate treatment regimen. The objective of this study was to examine the gene expression profiles of SIRT3, HRAS, LSP1, SCUBE2 and AP2A2 in Iranian women with BC.A total of 136 patients including healthy controls were categorized into three groups based on the relapse of the disease. Expression of desired genes in formalin-fixed, paraffin embedded tissues collected from all groups of participants was analyzed via the RT PCR method. RNA extraction and cDNA synthesis were performed then real-time quantitative PCR was carried out. Gene expression analysis revealed that the expression of SIRT3 was equal among patient and control groups. LSP1 was down regulated in all patient groups relative to controls but reduced expression in the metastatic group relative to the non-metastatic one was not significant. HRAS was significantly overexpressed in total and metastatic tumor samples versus normal but not in non-metastatic cases. SCUBE2 expression showed significant over-expression in both overall tumor samples and the non-metastatic group as compared to normal tissues. Gene expression level of AP2A2 in all groups was not detectable. Our data are compatible with a tumor suppressor role of LSP1 related to potential prognostic factor for tumor recurrence and outcome. This study for the first time assayed the prognostic value and changes in the expression of SIRT3, LSP1, HRAS, SCUBE2 and AP2A2 genes in women with breast cancer in the Iranian population and findings confirmed potential biomarker and prognostic capability of these genes. Such expression profiling data can critically improve prognosis and treatment decisions in cancer patients.
\end{abstract}

Keywords: : Breast cancer - gene profiling - SCUBE2 - SIRT3 - HRAS - LSP1 - AP2A2

Asian Pac J Cancer Prev, 17, Cancer Control in Western Asia Special Issue, 155-160

\section{Introduction}

Breast cancer $(\mathrm{BC})$ is one of the most common forms of cancers in Iran and is becoming the cause of most cancer-related deaths among Iranian women (Taghavi et al., 2012). The age-standardized incidence rate of cancer in 2009 was $28.3 \%$ for women and $0.8 \%$ in men. Precise diagnosis and early prediction of prognosis are central to any successful treatment of cancers. Recent years, there has been a rise in the use of multigene expression assays for predicting $\mathrm{BC}$ prognosis. This is because multigene expression profiling provides highly specific information and complements standard clinical practices and histopathological techniques, supporting clinicians in making critical treatment decisions (Patani et al., 2013).

$\mathrm{BC}$ is a complex disease involving genetic and environmental factors. In addition to various hormonal changes and lifestyle factors, genetic factors strongly contribute to the risk of developing cancers (Cancer, 2001; Cancer, 2002; Easton, 2002). Compared to those with no family history, women with a first-degree relative affected by $\mathrm{BC}$ are at an estimated two-fold increased risk of $\mathrm{BC}$ (Cancer, 2001).

New molecular techniques have allowed detection of genome-wide changes in cancer and have identified several genes responsible for tumor progression. We studied five genes, SIRT3, HRAS, LSP1, SCUBE2, and AP2A2, located on the chromosome 11p15. Although these genes were shown to play important roles in $\mathrm{BC}$ recurrence, their role in the recurrence of $\mathrm{BC}$ in Iranian population has not been established.

SIRT3 induces fatty acid oxidation during fasting in

${ }^{1}$ Department of Medical Genetics, ${ }^{2}$ Department of Pathology, Faculty of Medicine, ${ }^{6}$ Genomic Research Center, Shahid Beheshti University of Medical Sciences, ${ }^{3}$ Young Researcher and Elite Club, ${ }^{4}$ Department of Biology, Islamic Azad University, East Tehran Branch,Tehran, ${ }^{5}$ Department of Pharmaceutical Nanotechnology, School of Pharmacy, Zanjan, Iran. *For correspondence: v.yassaee-grc@sbmu.ac.ir 
hepatocytes via deacetylation of LCAD and targets the mitochondrial enzymes IDH2 and MnSOD (Someya et al.; Hirschey et al., 2010; Qiu et al., 2010) that play key roles in the maintenance of redox balance. Loss of SIRT3 has been reported to result in increase in cellular reactive oxygen species levels and contributes to numerous pathologies, including hearing loss and tumorigenesis (Kim et al., 2010).

A number of studies have investigated the association of HRAS alleles with incidence of cancers. However, the overall results have been inconclusive. While some studies found a statistically significant relationship between HRAS alleles and BC (Fornaro et al., 2013; Saini et al., 2013), others found either a negative, or a positive (Li-Weber, 2013a), but statistically insignificant association (Young et al., 2013; Giltnane and Balko, 2014). Activating mutations in the RAS genes, mainly in codons 12,13 , and 61 , occur in approximately $20 \%$ of all human cancers (Giltnane and Balko, 2014). Among these, mutations in KRAS account for approximately $85 \%$ of all RAS mutations found in human tumors, whereas that in NRAS and HRAS accounts for approximately $15 \%$ and $1 \%$ respectively (Li-Weber, $2013 \mathrm{~b}$ ).

In humans, the full-length transcript of a-adaptin subunit of AP2A2 is expressed in the forebrain, skeletal muscle, spinal cord, cerebellum, salivary gland, heart and colon (Scorilas et al., 2002). There are two a-adaptin genes, namely alpha A and alpha C (Buroker et al., 2011). During translation the alpha A mRNA is alternatively spliced resulting in two isoforms of the protein that are found in most tissues. The alpha $\mathrm{C}$ is predominantly expressed in brain. The shorter alpha A isoform lacks 21 amino acids that form part of the hinge region and shows similar electrophoretic mobility to that of alpha C (Scorilas et al., 2002). Transcripts of the shorter alpha A isoform and alpha $\mathrm{C}$ are found in heart tissue (Scorilas et al., 2002).

The gene SCUBE2 is an important prognostic marker of BC. SCUBE2 is linked to the expression of estrogen receptor $\alpha(E R \alpha)$. Searching for effective molecular markers is necessary to predict the disease course and guide treatment decision (Yang et al., 2002; Brennan and Gallagher, 2008). SCUBE2 is expressed in endothelium, fibroblasts, and renal mesangial cells (Yang et al., 2002; Fan et al., 2006). Although the precise function of SCUBE2 in these cell types is unknown, studies have shown its expression in primary invasive breast tumors (Cheng et al., 2009). Additionally, clinical studies have shown that patients with positive SCUBE2 protein-expression in tumors had better prognosis than those with tumors that lacked the expression of this protein (Cheng et al., 2009).

LSP1 encodes a lymphocyte specific intracellular Ca2+-binding phosphor protein. The LSP1 protein contains a basic C-terminal domain, which is highly conserved between mouse and human (Hannigan et al., 2001). Over expression of LSP1 increases the production of F-actin bundles which is likely to be responsible for the morphological abnormalities and cell migration defects. Hannigan et al. reported that reduced chemotactic responses of LSP1 neutrophils are associated with discontinuous primary actin-rich cortexes and large abnormal membrane protrusions (Howard et al., 1998). Overexpression of LSP1 causes morphologic and motile abnormalities characteristic of the NAD 47/89 phenotype (McKinney et al., 2010).

Although the functional roles of these genes in the development of immune system disorders and breast cancer are well documented (Howard et al., 1998; Hannigan et al., 2001; Fan et al., 2006; Cheng et al., 2009; McKinney et al., 2010), whether or not they contribute to increased $\mathrm{BC}$ risk in populations of non-European descent has not been investigated. In the present study, we investigated the expression of LSP1, HRAS, SIRT3, AP2A2 and SCUBE2 in Iranian BC patients and healthy control subjects.

\section{Materials and Methods}

\section{Patients and their characteristics}

Patients were recruited following informed, written consent using a process approved by the ethical committee of Deputy for Research affairs at Shahid Beheshti University of Medical Sciences, Tehran, Iran (Project number 237M) that followed the tenets of the Declaration of Helsinki.

Pertaining to the current research and literature, a preliminary study was conducted to determine the expression profiling of five genes SIRT3, HRAS, LSP1, SCUBE2 and AP2A2 which were reported to have a significant association with breast cancer in a small group of lymph-node negative patients. Between the years 2009 and 2011, samples from 3820 patients were collected from the hospitals of Tehran.

Recurrence was determined by radiographic, and ultrasonography examination, bone scan, and pathologic evidence. Patient characteristics were extracted from clinical and pathologic records (Table 1).

To validate our findings we expanded the study with similar analysis on larger number of patients. The analysis included patients who developed recurrent $\mathrm{BC}$ within five years and patients who remained disease-free for at least five years.

Paraffin-embedded tissue samples from three groups of participants were used in this study. The first group contained samples of those who had no relapse even after 5-7 years of treatment. The second group of patient samples was from those who had relapse after 5-7 years and the third group contained samples from those who underwent mammoplasty surgery for non-breast cancer related reasons. A total of 111 samples from BC cases and 20 cancer-free control samples were included in this study.

All patients were characterized as Iranian and no division into ethnic groups was performed. Informed written consent was obtained from all subjects who participated in this study.

\section{Gene expression analyses}

Total RNA was extracted from the FFPE tissue samples using the (QIAamp, USA) and the quality of RNA was evaluated with Agilent RNA 6,000 Nano Kit. Total RNA extracted was then reverse transcribed in a reaction 
Table 1. Characteristic Features of Metastatic and Non-Metastatic Groups

\begin{tabular}{lllllllllllllllllll}
\hline Age & & $\begin{array}{l}\text { Tumor Size } \\
\text { (Mean) }\end{array}$ & Lymph node & $\begin{array}{l}\text { Hormone } \\
\text { therapy }\end{array}$ & $\begin{array}{l}\text { Chemo } \\
\text { therapy }\end{array}$ & $\begin{array}{l}\text { Radio } \\
\text { therapy }\end{array}$ & Disease stage & ER & PR & Her-2 & Grade \\
\hline$\geq 50$ & $<50$ & $2.13(\mathrm{~mm})$ & $-\mathrm{ve}$ & $+\mathrm{ve}$ & Yes & No & Yes & No & Yes & No & 1 & 2 & $+/+$ & $+/-$ & + & - & 1 & 2 \\
57 & 41 & - & 111 & 0 & 111 & 0 & 111 & 0 & 111 & 0 & 57 & 41 & 38 & 60 & 73 & 25 & 57 & 41 \\
\hline
\end{tabular}

(Total number of samples=111); ER= Estrogen Receptor; PR= Progesterone Receptor; $+\mathrm{ve}=$ Positive; $-\mathrm{ve}=$ Negative.

mixture containing 250 mMTris- $\mathrm{HCl}$ buffer, $(\mathrm{pH} 8.3$ containing 375, $\mathrm{mMKCl}$, and $15 \mathrm{mM} \mathrm{MgCl2}$ ) (Applied Biosystems, USA), 0.1 M DTT (Applied Biosystems, USA), 10 mMdNTPs (Fermentas, USA), 20 U/reaction of RNasin ${ }^{\mathrm{TM}}$ ribonuclease inhibitor (Applied Biosystems, USA) and $200 \mathrm{U} /$ reaction of Superscript ${ }^{\mathrm{TM}}$ III RT (Applied Biosystems, USA). The cDNA thus obtained was diluted 10 -fold in $2 \mathrm{ng} / \mu \mathrm{L}$ polyinosinic acid and used in quantitative PCR (qPCR) reactions.

\section{Real time quantitative PCR ( $q P C R)$}

The qPCR reactions were performed in 96-well plates on a Real Time PCR 7500 (Applied Bio System) instrument. Typically, a total $20 \mu \mathrm{L}$ of the reaction mixture contained $100 \mathrm{ng}$ of cDNA, $12.5 \mu \mathrm{L}$ of TaqMan universal PCR master mix (Applied Bio System), $1 \mu \mathrm{L}$ each of $10 \mathrm{mmol} / \mu \mathrm{L}$ forward and reverse primers, and $4.5 \mu \mathrm{L}$ nuclease-free water. The relative gene expression levels were determined using the $(\Delta \Delta \mathrm{ct})$ method. All analyses were replicated. The relative gene expression levels were determined using the delta delta $\mathrm{Ct}$ method.

The thermal cycling conditions consisted of an initial denaturation at $95^{\circ} \mathrm{C}$ for $10 \mathrm{~min}$, followed by 45 cycles of denaturation at $95^{\circ} \mathrm{C}$ for $15 \mathrm{~s}$, annealing at $60^{\circ} \mathrm{C}$ for $1 \mathrm{~min}$. To minimize experimental variability of the $\mathrm{Ct}$ values, the threshold cycle where the fluorescence signal rises significantly above background in the exponential phase of the amplification was determined by the second derivative maximum method. Genes were selected from gene expression databases; GAPDH was selected as a negative control. The probes and primers were designed based on the conserved regions of genes using the primer express software (Life Technologies-ABI). In order to compare RNA extraction results with those of the real-time PCR, RNA isolation and RT-PCR experiments were repeated one to two times.

\section{Results}

To evaluate the relative expression levels of SIRT3, SCUBE2, HRAS, AP2A2, and LSP1, all 111 tumor samples and 20 healthy controls were analyzed by qPCR. GAPDH was used as an internal control.

Expression analysis of genes and the respective control group are described below:

Gene expression analysis using Real-Time PCR revealed the expression of SIRT3 in all 111 patients to be approximately equal with that of healthy control women. However, a distinct down regulation in non-metastatic group (fold change $=0.9$ ) and up regulation in metastatic group (fold change $=1.4$ ) were observed which were statistically insignificant. Comparative expression analysis

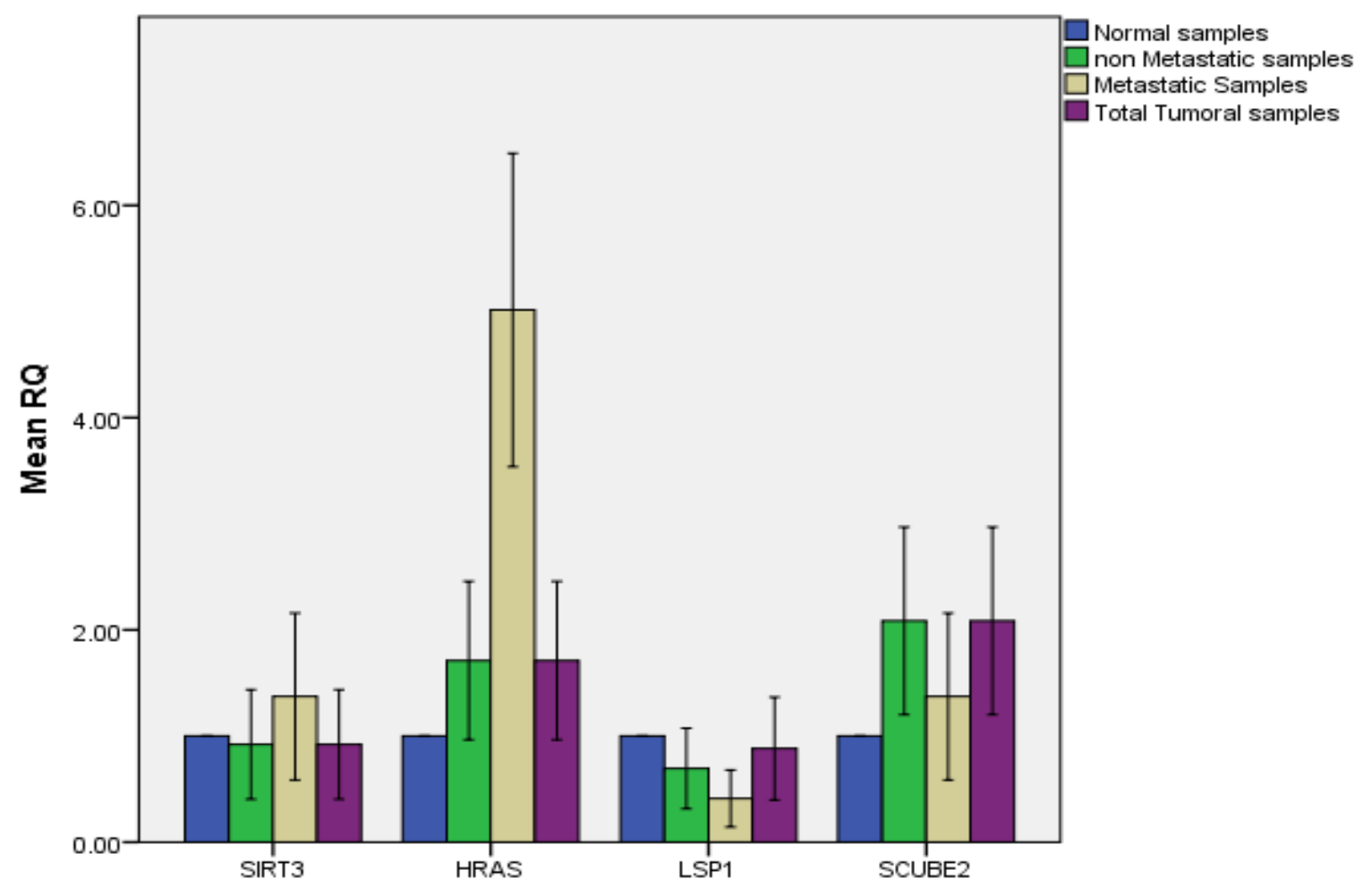

Figure 1. Q-PCR Results in Normal, Non-Metastatic, Metastatic and Total Patients Groups for the Specified Genes 
among metastatic and non-metastatic groups showed a 1.58 fold overexpression in the metastatic relative to non-metastatic group; nonetheless, the difference was not statistically significant. HRAS showed considerable overexpression in tumor samples when compared to normal (fold change $=2.5$, p-value $<0.01$ ), whereas analysis of the individual groups determined a significant overexpression of this gene in the metastatic with mean fold of 5 (p-value $<0.01$ ) and an insignificant 1.6 fold overexpression of non-metastatic groups in comparison to that of controls. HRAS gene expression analysis in metastatic relative to non-metastatic samples showed 3.9 fold overexpression ( $\mathrm{p}$-value $<0$. 01). LSP1 down regulated in both non-metastatic and metastatic cases as to normal samples with fold changes $(0.7, \mathrm{P}$-value $<$ $0.01)$, and $(0.5, p$-value $<0.01)$ respectively. Therefore, relative expression of total tumor samples revealed reduction in expression (fold change $=0.6, \mathrm{p}$-value $<0.01)$. An insignificant expression value $(0.7)$ of LSP1 was determined in metastatic group relative to non-metastatic samples. SCUBE2 expression analysis determined a significant over expression in both total and non-metastatic samples as to the healthy controls with RQ of 1.96 and 2.3 respectively but expression in metastatic group versus controls was not significant $(\mathrm{RQ}=1.18)$. Comparison of expression in two metastatic and non-metastatic groups revealed a significant 1.9 fold overexpression in the metastatic group $(p<0.01)$. Gene expression level of AP2A2 in all samples was undetectable.

Signature profiles of the genes SIRT3, SCUBE2, HRAS, and LSP1 in the two metastatic and non-metastatic groups determined HRAS and LSP1 with the highest and lowest in the metastatic group respectively while in the non-metastatic group SCUBE2 and LSP1 genes showed the highest and lowest expressions respectively. Also statistical analysis in total samples showed the same result as non-metastatic group (Figure 1).

In summary, our results totally revealed over expression in SIRT3 and HRAS; however, LSP1 and SCUBE2 showed down regulation.

\section{Discussion}

Genome-wide association studies (GWAS) has identified abundant loci and SNPs with significant association to breast cancer susceptibility in the general population (Jiang et al., 2011). In 2007, Easton et al. and Stacey et al (Easton et al., 2007; Stacey et al., 2007) identified three breast cancer susceptibility loci at 8q24, $2 q 35$ and 11 p15 in the vicinity of LSP1 gene. According to the current studies we evaluated chromosome 11p15 to determine the association of the cancer genes with the overall risk of breast cancer in the Iranian population. To determine the expression profile of the genes and risk of early metastases in lymph node-negative patients who have been earlier treated for $\mathrm{BC}$, five genes named SIRT3, HRAS, LSP1, SCUBE2 and AP2A2 which may associate to $\mathrm{BC}$ were selected. All the patients with lymph node-negative received adjuvant chemotherapy or hormonal therapy; therefore, we could not evaluate the prognostic profile in lymph node-negative patients who did not receive any treatment for the disease. However, no influence of adjuvant chemotherapy was observed on the prognostic profiles (data not shown).

Mutations in the RAS genes were reported in approximately $20 \%$ of all human cancers (Irahara et al., 2010). Among these, $85 \%$ of the mutations are found in KRAS, whereas NRAS and HRAS mutations are reported in $15 \%$ and $1 \%$ of cancers, respectively. The RAS genes are expressed in tissues with a specific manner. HRAS is highly expressed in skin and skeletal muscles, KRAS expressed in colon and thymus and NRAS in male germinal tissue (Membrino et al., 2011). HRAS mutations are less common, but they have a high prevalence in skin papilloma's and urinary bladder tumors. More than half of bladder tumors have increased expression of HRAS and both mutations and over expression of the gene can contribute to bladder cancer (Schubbert et al., 2007). Additionally, it has recently been shown that the constitutively active HRAS expressed at low levels that can induce urothelial hyperplasia, whereas at high expression level, it makes rapidly growing and penetrating tumors throughout the urinary tract (Membrino et al., 2011).

In this study, the expression level of HRAS in breast tissue was evaluated in lymph node-negative patients with a risk of recurrence of breast cancer. Evaluations revealed an increased gene expression in both metastatic and non-metastatic groups in comparison to the healthy controls; however, the approximately 4 fold increase in the metastatic group suggests the function of this gene on tumor growth and metastatic features (Özer et al., 2000).

The LSP1 gene was originally isolated from T-lymphocytes and is considered to play a role in trans-membrane signaling. LSP1 is down regulated in apoptotic cells, and thus likely that over expression of this gene can induce resistance to apoptosis (Antoniou et al., 2009). The expression level of LSP1 along with its impact on clinical, pathological characteristics and prognosis on $\mathrm{BC}$ development were studied (Antoniou et al., 2009). The expression level of LSP1 in BC was significantly associated with positive ER and PR status; however, no other significant links with patient or tumor characteristics were observed (Antoniou et al., 2009). We determined significant down regulation of this gene in both groups. Although the function of this gene is not well defined, our results suggest that LSP1 may act as a tumor suppressor gene.

SCUBE2 is expressed in osteoblasts and vascular cells (Tsai et al., 2009). Although some existing data suggest that SCUBE2 can act as a novel breast tumor suppressor gene, the specific protein domain responsible for its tumor suppressor effect or the mechanisms of its anti-tumor activity is not well defined (Lin et al., 2011). However, SCUBE2 expression was also detected in primary invasive breast tumors (Lin et al., 2011). Maciej et al examined the expression of SCUBE2 in normal and malignant samples and its association with PTEN and steroid hormone receptors. They reported that in epithelial cancer, SCUBE2 expression is reduced in G3 stage compared with G1 stage or in postmenopausal (Skrzypczak et al., 2013). Our study determined a considerable upregulation of the 
SCUBE2 in the non-metastatic samples in comparison to the metastatic group supporting the findings of Lin YC et al demonstrating that SCUBE2 acts as a tumor suppressor gene(Lin et al., 2014). Over expression of SCUBE2 will effectively increase the E-cadherin-b-catenin junctions to promote epithelial differentiation and inhibit invasion of carcinoma cells by epithelial-mesenchymal transition (EMT) reversal(Lin et al., 2014). Clinical studies with anti-SCUBE2 immunohistochemistry revealed that SCUBE2 positive patients had better chances of survival than SCUBE2 negative patients (Cheng et al., 2009). Therefore, SCUBE2 acts as a novel tumor suppressor gene and an effective prognostic marker.

Ashraf and colleagues (2006) reported greater expression of SIRT3 gene in lymph node-positive breast cancer patients in comparison with healthy controls. Mitochondria houses SIRT3 within the inner mitochondrial membrane cristae and the matrix (Shi et al., 2005; Ashraf et al., 2006) where it functions in a wide range of activities including metabolism, prevention of apoptosis, aging and senescence (Vaziri et al., 2001). Here we observed a drastic decrease of SIRT3 in both non-metastatic and metastatic groups in comparison to healthy controls; however, the downregulation was more significant in the non-metastatic group. In other words, SIRT3 expression levels were higher in the metastatic group as to the non-metastatic cohort. Over expression of SIRT3 in the metastatic samples may suggest its contribution to survival and dissemination of aggressive tumors (Kincaid and Bossy-Wetzel, 2013).

By using microarray gene expression analysis of breast cancer samples, Chou and coworkers have shown significant differences in the expression of AP2A2 gene along with 21 other genes(Chou et al., 2013). However, our study did not reveal any significant changes in the expression pattern of AP2A2 gene between metastatic, non-metastatic and control groups. Our data serves as a potential prognosis signature denoting that patients with lymph node-negative disease may be at a high risk for development of metastatic breast cancer.

In summary, our findings support clinical utilization of LSP1, HRAS, AP2A2 and SCUBE2 as independent prognostic markers for breast cancer recurrence. Alterations in expression of these genes play an important role in differentiation of breast cancer cells and tumor progression. Prognosis profile is a strong predictor of the development of metastases in patients with lymph node-negative disease. It is noteworthy to report, since the presence of lymph-node metastasis by itself is a strong predictor of poor survival. Providing an expression profile for breast cancer patient may lead to predict BC development earlier as well as selective treatment and effective prognosis.

\section{Acknowledgements}

We thank the patients and their families for their cooperation and participation. The study was funded by Genomic Research Center at Shahid Beheshti University of Medical Sciences, Tehran, Iran. The authors declare no conflict of interest and no financial competing interests.

\section{References}

Antoniou AC, Sinilnikova OM, McGuffog L, et al (2009). Common variants in LSP1, 2q35 and 8q24 and breast cancer risk for BRCA1 and BRCA2 mutation carriers. Hum $\mathrm{Mol}$ Genet, 18, 4442-56.

Ashraf N, Zino S, MacIntyre A, et al (2006). Altered sirtuin expression is associated with node-positive breast cancer. Br J Cancer, 95, 1056-61.

Brennan DJ, Gallagher WM (2008). Prognostic ability of a panel of immunohistochemistry markers - retailoring of an 'old solution'. Breast Cancer Res, 10, 102.

Buroker NE, Huang J-Y, Barboza J, et al (2011). The adaptor-related protein complex 2, alpha 2 subunit (AP2 $\alpha 2$ ) gene is a peroxisome proliferator-activated receptor cardiac target gene. Protein $J, \mathbf{3 1}, 75-83$.

Cancer CGoHFiB (2001). Familial breast cancer: collaborative reanalysis of individual data from 52 epidemiological studies including 58209 women with breast cancer and 101986 women without the disease. Lancet, 358, 1389-99.

Cancer CGoHFiB (2002). Alcohol, tobacco and breast cancer - collaborative reanalysis of individual data from 53 epidemiological studies, including 58515 women with breast cancer and 95067 women without the disease. $\mathrm{Br} \mathrm{J}$ Cancer, 87, 1234-45.

Cheng CJ, Lin YC, Tsai MT, et al (2009). SCUBE2 suppresses breast tumor cell proliferation and confers a favorable prognosis in invasive breast cancer. Cancer Res, 69, 3634-41.

Chou HL, Yao CT, Su SL, et al (2013). Gene expression profiling of breast cancer survivability by pooled cDNA microarray analysis using logistic regression, artificial neural networks and decision trees. BMC Bioinformatics, 14, 1-11.

Easton DF (2002). Familial risks of breast cancer. Breast Cancer Res, 4, 1-3.

Easton DF, Pooley KA, Dunning AM, et al (2007). Genome-wide association study identifies novel breast cancer susceptibility loci. Nature, 447, 1087-93.

Fan C, Oh DS, Wessels L, et al (2006). Concordance among Gene-Expression-Based Predictors for Breast Cancer. $N$ Engl J Med, 355, 560-9.

Fornaro L, Lonardi S, Masi G, et al (2013). FOLFOXIRI in combination with panitumumab as first-line treatment in quadruple wild-type (KRAS, NRAS, HRAS, BRAF) metastatic colorectal cancer patients: a phase II trial by the Gruppo Oncologico Nord Ovest (GONO). Ann Oncol, 24, 2062-7.

Giltnane JM, Balko JM (2014). Rationale for targeting the Ras/ MAPK pathway in triple-negative breast cancer. Discov Med, 17, 275-83.

Hannigan M, Zhan L, Ai Y, et al (2001). Leukocyte-specific gene 1 protein (LSP1) is involved in chemokine $\mathrm{KC}$-activated cytoskeletal reorganization in murine neutrophils in vitro. J Leukoc Biol, 69, 497-504.

Hirschey MD, Shimazu T, Goetzman E, et al (2010). SIRT3 regulates mitochondrial fatty-acid oxidation by reversible enzyme deacetylation. Nature, 464, 121-5.

Howard TH, Hartwig J, Cunningham C (1998). Lymphocyte-specific protein 1 expression in eukaryotic cells reproduces the morphologic and motile abnormality of NAD 47/89 neutrophils. Blood, 91, 4786-95.

Irahara N, Baba Y, Nosho K, et al (2010). NRAS mutations are rare in colorectal cancer. Diagn Mol Pathol, 19, 157-63.

Jiang Y, Shen H, Liu Xa, et al (2011). Genetic Variants at 1p11.2 and Breast Cancer Risk: A Two-Stage Study in Chinese Women. PLoS ONE, 6, e21563.

Kim HS, Patel K, Muldoon-Jacobs K, et al (2010). SIRT3 Is a mitochondria-localized tumor suppressor required for maintenance of mitochondrial integrity and metabolism during 
Stress. Cancer Cell, 17, 41-52.

Kincaid B, Bossy-Wetzel E (2013). Forever young: SIRT3 a shield against mitochondrial meltdown, aging, and neurodegeneration. Front Aging Neurosci, 5, 48.

Li-Weber M (2013a). Targeting apoptosis pathways in cancer by Chinese medicine. Cancer Lett, 332, 304-12.

Li-Weber M (2013b). Targeting apoptosis pathways in cancer by Chinese medicine. Cancer Lett, 332, 304-12.

Lin Y-C, Chen C-C, Cheng C-J, et al (2011). Domain and functional analysis of a novel breast tumor suppressor protein, SCUBE2. J Biol Chem, 286, 27039-47.

Lin YC, Lee YC, Li LH, et al (2014). Tumor suppressor SCUBE2 inhibits breast-cancer cell migration and invasion through the reversal of epithelial-mesenchymal transition. J Cell Sci, 127, 85-100.

McKinney C, Fanciulli M, Merriman ME, et al (2010). Association of variation in $\mathrm{Fc} \gamma$ receptor $3 \mathrm{~B}$ gene copy number with rheumatoid arthritis in Caucasian samples. Ann Rheum Dis, 69, 1711-6.

Membrino A, Cogoi S, Pedersen EB, et al (2011). G4-DNA formation in the HRAS promoter and rational design of decoy oligonucleotides for cancer therapy. PLoS ONE, 6 , e24421.

Özer E, Sis B, Özen E, et al (2000). BRCA1, C-erbB-2, and $\mathrm{H}$-ras gene expressions in young women with breast cancer: an immunohistochemical study. Appl Immunohistochem Mol Morphol, 8, 8-12.

Patani N, Martin LA, Dowsett M (2013). Biomarkers for the clinical management of breast cancer: international perspective. Int $J$ Cancer, 133, 1-13.

Qiu X, Brown K, Hirschey MD, et al (2010). Calorie restriction reduces oxidative stress by SIRT3-mediated SOD2 Activation. Cell Metab, 12, 662-7.

Saini KS, Loi S, de Azambuja E, et al (2013). Targeting the PI3K/ AKT/mTOR and Raf/MEK/ERK pathways in the treatment of breast cancer. Cancer Treat Rev, 39, 935-46.

Schubbert S, Shannon K, Bollag G (2007). Hyperactive Ras in developmental disorders and cancer. Nat Rev Cancer, 7, 295-308.

Scorilas A, Levesque MA, Ashworth LK, et al (2002). Cloning, physical mapping and structural characterization of the human $\alpha \mathrm{A}$-adaptin gene. Gene, 289, 191-9.

Shi T, Wang F, Stieren E, et al (2005). SIRT3, a mitochondrial sirtuin deacetylase, regulates mitochondrial function and thermogenesis in brown adipocytes. J Biol Chem, 280, 13560-7.

Skrzypczak M, Lattrich C, Häring J, et al (2013). Expression of SCUBE2 gene declines in high grade endometrial cancer and associates with expression of steroid hormone receptors and tumor suppressor PTEN. Gynecol Endocrinol, 29, 1031-5.

Someya S, Yu W, Hallows WC, et al (2012). Sirt3 mediates reduction of oxidative damage and prevention of age-related hearing loss under Caloric restriction. Cell, 143, 802-12.

Stacey SN, Manolescu A, Sulem P, et al (2007). Common variants on chromosomes $2 \mathrm{q} 35$ and $16 \mathrm{q} 12$ confer susceptibility to estrogen receptor-positive breast cancer. Nat Genet, 39, 865-9.

Taghavi A, Fazeli Z, Vahedi M, et al (2012). Increased Trend of Breast Cancer Mortality in Iran. Asian Pac J Cancer Prev, 13, 367-70.

Tsai M-T, Cheng C-J, Lin Y-C, et al (2009). Isolation and characterization of a secreted, cell-surface glycoprotein SCUBE2 from humans. Biochem J, 422, 119-28.

Vaziri H, Dessain SK, Eaton EN, et al (2001). em hSIR2 sup SIRT1 sup em Functions as an NAD-Dependent p53 Deacetylase. Cell, 107, 149-59.

Yang R-B, Ng CKD, Wasserman SM, et al (2002). Identification of a novel family of cell-surface proteins expressed in human vascular endothelium. $J$ BiolChem, 277, 46364-73.

Young A, Lou D, McCormick F (2013). Oncogenic and wild-type ras play divergent roles in the regulation of mitogen-activated protein kinase signaling. Cancer Discov, 3, 112-23. 